\title{
Intérêt de l'oxygénothérapie hyperbare dans la prévention et le traitement des ostéonécroses des maxillaires dues aux bisphosphonates
}

\author{
Mathieu Gunepin ${ }^{1}{ }^{*}$, Florence Derache ${ }^{2}$, Olivier Cathelinaud ${ }^{3}$, Jean-Pierre De Jaureguibery ${ }^{4}$, \\ Jean-Sébastien Bladé ${ }^{4}$, Olivier Gisserot ${ }^{3}$, Jean-Jacques Risso ${ }^{5}$, Jean-Eric Blatteau ${ }^{5}$, \\ Michel Hugon ${ }^{6}$, Benoît Bédrune ${ }^{2}$, Didier Rivière ${ }^{1}$ \\ ${ }^{1}$ Centre médical des Armées, Draguignan, France \\ 2 Service de Chirurgie maxillo-faciale et Stomatologie, Hôpital d'Instruction des Armées Sainte Anne, Toulon, France \\ 3 Service d'ORL et de Chirurgie cervico-faciale, Hôpital d'Instruction des Armées Sainte Anne, Toulon, France \\ ${ }^{4}$ Service de Médecine interne-Oncologie, Hôpital d'Instruction des Armées Sainte Anne, Toulon, France \\ ${ }^{5}$ Equipe résidante de Recherche subaquatique opérationnelle, Institut de Recherche biomédicale des Armées, Toulon, France \\ ${ }^{6}$ Service de Médecine hyperbare et Expertise de la plongée, Toulon, France
}

(Reçu le 25 octobre 2012, accepté le 3 décembre 2012)

Mots clés : bisphosphonates / ostéonécrose des maxillaires / oxygénothérapie hyperbare / ostéoporose / cancer

Key words: bisphosphonates / osteonecrosis of the jaws / hyperbaric oxygen therapy / osteoporosis / cancer

\begin{abstract}
Résumé - Les bisphosphonates (BP) sont des inhibiteurs du remodelage osseux indiqués dans la prise en charge de pathologies malignes (myélome multiple, métastases de tumeurs solides, etc.) et bénignes (ostéoporose, maladie de Paget, etc.). Depuis 2003, un effet indésirable imputé à cette classe de médicaments est apparu dans la littérature : les ostéonécroses des maxillaires (ONM) induites par la prise de BP. Cliniquement, l'ONM se caractérise par une exposition d'os nécrotique qui persiste pendant plus de 8 semaines. En 2007, puis 2009, l'American Association of Oral and Maxillofacial Surgeons (AAOMS) a émis des recommandations destinées aux professionnels de santé pour prévenir et prendre en charge cet effet indésirable. Cependant, l'absence de thérapeutiques efficaces a poussé de nombreux auteurs à tester, en complément des thérapies proposées par l'AAOMS, des traitements expérimentaux au nombre desquels figure l'oxygénothérapie hyperbare (OHB). D'un point de vue théorique, l'étiopathogénie des ONM n'étant pas connue, l'impact de l’OHB sur les mécanismes de survenue de cette pathologie reste hypothétique. D'un point de vue clinique, les avis divergent sur l'intérêt de l'utilisation de l'OHB dans le traitement des ONM. Cependant, les résultats préliminaires de deux essais cliniques contrôlés et randomisés en cours aux Etats-Unis laissent à penser que l'OHB pourrait trouver de nouvelles indications en chirurgie orale dans le traitement mais aussi la prévention des ONM.
\end{abstract}

\begin{abstract}
Interest of hyperbaric oxygen therapy in the prevention and treatment of bisphosphonate-related osteonecrosis of the jaws. Bisphosphonates (BP) have been used since 1960s for their capability to alter the mechanism of bone resorption and remodeling. Intravenous (IV) BP are effective in the treatment and management of cancer-related conditions including hypercalcemia of malignancy, skeletal-related events associated with bone metastases in the context of solid tumors such as breast cancer, prostate cancer and lung cancer, and management of lytic lesions in the setting of multiple myeloma. Oral BP are used to treat osteoporosis and osteopenia. In 2003, oral and maxillofacial surgeons first recognized and reported cases of non-healing exposed bone in the maxillofacial region in patients treated with IV bisphosphonates. This adverse effect was called BONJ for bisphosphonate-related osteonecrosis of the jaws. Clinically, BONJ is defined by an exposed bone in the maxillofacial region that has persisted for more than 8 weeks. So far, the etiopathogenesis of BONJ remains
\end{abstract}

*Correspondance : mgunepin@yahoo.fr 
uncertain. It is worth noting that BP act at the following levels: physicalchemical, tissue, cellular, and molecular. In 2007, then in 2009, strategies for management of patients with or at risk for BONJ were set forth in the American Association of Oral and Maxillofacial Surgeons (AAOMS) Position Paper on BONJ. BONJ's treatment comprises the following: pain control, antibiotic therapy, mouth rinse, BP discontinuation, and surgical debridement. Such measures, however, do not always achieve the resolution of the clinical findings. That led numerous authors to suggest, in complement to treatments advised by AAOMS, many experimental treatments as hyperbaric oxygen therapy (HBOT). HBOT is a way of treatment which increases the oxygen content of the blood and, consequently, the amount of oxygen delivered to tissues. The proposed rationale behind the beneficial effects of HBOT in ONM is increased wound healing, reduction of oedema and inflammation, stem cell mobilization and moderation of the suppression of bone turnover by BP. At the moment there is a controversy concerning the effectiveness of HBOT in the treatment of BONJ. But there are two ongoing randomized controlled trials in USA which are testing specifically the effectiveness of adjunctive HBOT in the management of BONJ. Although early results are encouraging, evidence-based conclusions could be made after the final results of these studies are published.

Les bisphosphonates (BP) sont utilisés dans la prise en charge de pathologies malignes (myélome multiple, métastases de tumeurs solides et hypercalcémie maligne) ou bénignes (ostéoporose, maladie de Paget, etc.) pour leur capacité à inhiber la résorption osseuse [1]. Avec l'augmentation de l'utilisation des BP et de leur durée de prescription, les premiers effets indésirables induits par la prise de BP ont été rapportés. Les plus courants sont l'oesophagite et les myalgies $[2,3]$. En 2003, 36 cas d'ostéonécrose des maxillaires (ONM) ont été rapportés chez des patients traités par zolédronate ou pamidronate [4]. Depuis, les ONM ont été reconnues comme un effet indésirable lié à la prise de $\mathrm{BP}$, avec un impact potentiellement délétère sur la qualité de vie des patients [4].

Les signes cliniques, les symptômes, l'étiopathogénie, les mesures préventives, les effets de l'arrêt des BP ainsi que les indicateurs d'évolution des ONM restent largement méconnus. De plus, la pertinence et l'efficacité des traitements des ONM actuellement utilisés restent sujettes à caution. En complément des thérapeutiques « classiques » conseillées par l'American Association of Oral and Maxillofacial Surgeons (AAOMS), de nombreux auteurs proposent des thérapeutiques dites expérimentales dont l'oxygénothérapie hyperbare.

\section{Bisphosphonates}

Les BP sont des analogues structuraux des pyrophosphates naturels au sein desquels l'atome d'oxygène central a été remplacé par un atome de carbone [5]. Selon que leur radical comporte ou non un atome d'azote, on distingue deux groupes de $B P$ : les $B P$ sans amine dits de première génération et les amino-BP qui représentent les $B P$ de deuxième et de troisième générations. La structure chimique des BP leur confère des propriétés utilisables en médecine, notamment celle de ralentir le remodelage osseux, principalement par inhibition de l'activité des ostéoclastes. Administrés par voie intraveineuse (IV), les BP sont indiqués dans la prévention des évènements osseux dans la prise en charge des myélomes multiples, de certaines tumeurs malignes avancées et des hypercalcémies malignes [5]. Les BP constituent par ailleurs le traitement le plus largement prescrit dans le cadre de la prise en charge de maladies bénignes comme l'ostéoporose post-ménopausique chez les femmes à haut risque de fracture, l'ostéoporose masculine et l'ostéoporose cortico-induite.

\section{ONM induites par la prise de BP}

\section{Définition}

En 2003, Wang et al. [6], puis Marx [7] décrivaient un nouveau type d'ONM extrêmement résistant aux thérapeutiques usuelles. Ces patients n'ayant pour point commun que la prise $\mathrm{de} \mathrm{BP}$, les auteurs concluaient à une association entre cette classe de médicaments et la survenue de ce qu'on commença à appeler les ONM. Actuellement, les critères diagnostiques internationalement reconnus d'une ONM sont $[8,9]$ :

- une lésion de la muqueuse dans la région maxillo-faciale mettant à nu l'os nécrosé et persistant depuis plus de 8 semaines,

- un traitement par BP antérieur ou en cours,

- une absence d'antécédents de radiothérapie dans la région maxillaire et

- une absence de localisation métastatique dans la zone de I'ONM confirmée par la réalisation systématique d'un examen histopathologique $[10,11]$.

\section{Classification des ONM}

L'AAOMS a défini en 2007 différents stades évolutifs, en fonction de l'aspect clinique afin d'établir des stratégies thérapeutiques adaptées [2,9]. Trois stades sont proposés (cf. Tab. I). En 2009, l'AAOMS a ajouté un stade 0 à la classification des ONM $[9,12]$ afin de ne pas exclure les patients qui, ayant reçu des BP, présentent des symptômes non spécifiques, 
Tableau I. Classification des ONM et prise en charge thérapeutique proposées par l'AAOMS [2, 9]. Table I. Clinical staging of ONM and the treatment proposed by the AAOMS [2, 9].

\begin{tabular}{|c|c|c|}
\hline Groupe & Description & Stratégie thérapeutique \\
\hline A risque & $\begin{array}{l}\text { - patients traités ou ayant été traités par BP et ne } \\
\text { présentant pas d'os nécrotique exposé }\end{array}$ & $\begin{array}{l}\text { - information du patient sur les risques de survenue d'une ONM } \\
\text { - information sur la symptomatologie des ONM }\end{array}$ \\
\hline Niveau 0 & $\begin{array}{l}\text { - absence d'os nécrotique exposé } \\
\text { - signes cliniques non spécifiques }\end{array}$ & $\begin{array}{l}\text { - prise en charge systémique avec recours aux antibiotiques et } \\
\text { aux antalgiques }\end{array}$ \\
\hline Niveau 1 & $\begin{array}{l}\text { - os nécrotique exposé } \\
\text { - patient asymptomatique sans signe clinique évident } \\
\text { d'infection }\end{array}$ & $\begin{array}{l}\text { - bains de bouche antiseptique à base de chlorexhidine } 0,12 \% \\
\text { - réévaluation de la poursuite du traitement si BP oraux }\end{array}$ \\
\hline Niveau 2 & $\begin{array}{l}\text { - os nécrotique exposé } \\
\text { - douleurs avec présence d'une infection } \\
\text { - érythème autour de la zone exposée avec ou sans } \\
\text { écoulement purulent }\end{array}$ & $\begin{array}{l}\text { - réalisation de rinçages locaux avec des antiseptiques } \\
\text { - réalisation d'un débridement osseux superficiel afin d'éviter } \\
\text { l'irritation des structures molles adjacentes par l'os séquestré } \\
\text { - prescription d'antibiotiques à large spectre } \\
\text { - prescription d'antalgiques }\end{array}$ \\
\hline Niveau 3 & $\begin{array}{l}\text { - os nécrotique exposé } \\
\text { - douleurs avec présence d'une infection } \\
\text { - présence de fractures pathologiques, de fistules } \\
\text { cutanées ou d'ostéolyse étendue }\end{array}$ & $\begin{array}{l}\text { - en plus de la prescription d'antiseptiques, d'antibiotiques et } \\
\text { d'antalgiques, il est préconisé de réaliser une résection } \\
\text { osseuse suivie d'une reconstruction mandibulaire }\end{array}$ \\
\hline
\end{tabular}

potentiellement liés à des modifications du métabolisme osseux induit par les BP :

- douleur dentaire sans cause identifiée pouvant être liée à des modifications du métabolisme osseux local,

- douleur du sinus maxillaire éventuellement associée à une inflammation loco-régionale,

- paresthésies ou dysesthésies provoquées par l'inflammation,

- douleurs neuropathiques qui peuvent parfois répondre à la gabapentine ou à une autre molécule de ce type.

\section{Incidence des ONM}

Les données publiées concernant l'incidence des ONM chez les patients atteints de pathologie maligne traités par BP IV mentionnent des incidences très variables (entre $0,8 \%$ et $12 \%$ ) [8]. Cependant, il est important de noter qu'il s'agit d'études rétrospectives portant sur de petits effectifs insuffisants [8] et que plusieurs études rapportent des incidences plus importantes, jusqu'à $28 \%$ [9, 13, 14]. Ces écarts pourraient résulter d'une approche méthodologique différente entre les études : durée de suivi des patients, modalités du dépistage des ONM, etc. [15]. Dans l'ostéoporose et la maladie de Paget, l'incidence des ONM sous BP per os est très mal connue. Toutefois, quelques publications mentionnent que le risque de survenue d'une ONM est bien plus faible que celui observé chez les patients traités par BP IV pour une pathologie maligne et serait estimée à 1 cas pour 10000 à 100000 patient-années $[8,16,17]$.

\section{Etiopathogénie}

L'étiopathogénie précise des 0 NM reste incertaine. Ceci est notamment la conséquence :

- de l'action multiple des BP à différents niveaux (physicochimique, tissulaire, cellulaire et moléculaire) [18-20],

- du nombre important de facteurs favorisant le risque de survenue des ONM (cf. Tab. II).

Pour expliquer le processus de survenue d'une ONM, plusieurs théories ont été proposées :

- théorie de l'hypocellularité : elle repose sur l'apoptose des cellules osseuses induites par les amino-BP qui conduirait à une diminution du remodelage osseux mais qui limiterait aussi les capacités de réparation et de cicatrisation du tissu osseux $[5,21]$.

- théorie de l'hypovascularisation : les effets anti-angiogéniques de certains BP, notamment le zolédronate, entraîneraient une diminution de la vascularisation osseuse avec survenue d'une ostéonécrose avasculaire [5, 22].

- théorie infectieuse : des traumatismes pourraient altérer la muqueuse buccale entraînant l'exposition du tissu osseux sousjacent hypovascularisé. Le contact de l'os avec le milieu buccal septique entraînerait une surinfection secondaire [5].

- théorie de la toxicité propre aux BP : les BP auraient un effet toxique sur l'épithélium buccal du fait de leur forte concentration dans l'os sous-jacent [23, 24].

\section{Traitements et prévention des ONM}

L'objectif principal de la prévention de survenue des ONM chez les patients à risque mais aussi du traitement des ONM 
Tableau II. Proposition d'utilisation de l'OHB pour la prévention et le traitement des ONM en fonction du risque de survenue et du stade d'évolution de l'ONM.

Table II. Proposition of use of HBOT for the prevention and treatment of BONJ according to the risk factors of occurrence and the clinical staging of BONJ.

Groupe de patient $\quad$ Protocole d'0HB

(séances de 2,5 à 2,8 ATA - 90 à 120 minutes)

Patient à risque
(traité ou ayant été traité par $\mathrm{BP})$
Patient avec ONM de stade 1

est de préserver la qualité de vie des patients, de contrôler la douleur et l'infection et d'éviter le développement de zone d'exposition de tissu osseux nécrotique dans la cavité buccale [25].

Traitements : recommandations (cf. Tab. I)

Il n'existe pas de traitement efficace pour les ONM [25]. La prescription d'antibiotiques à large spectre au long cours, de bains de bouche anti-microbiens, d'antalgiques si nécessaire et d'un suivi médical et dentaire rigoureux est recommandée chez les patients présentant des lésions asymptomatiques [2]. L'une des difficultés de la prise en charge des ONM est que l'approche chirurgicale, notamment la réalisation d'un débridement des tissus nécrotiques et la couverture de l'os exposé, conduit le plus souvent à un échec avec augmentation de la surface osseuse exposée dans la cavité buccale [20, 26-28].

Thérapeutiques expérimentales

- Utilisation du laser

Elle représente $14 \%$ des thérapeutiques expérimentales selon Filleul et al. [29]. Certains auteurs préconisent une approche chirurgicale dont le but est d'obtenir une fermeture muqueuse pérenne et donc un isolement de l'os par rapport à la cavité buccale, évitant ainsi les surinfections secondaires [5]. Dans ce cas, l'association d'un traitement conservateur au laser à la chirurgie d'exérèse donnerait les meilleurs résultats $[30,31]$. Les lasers sont utilisés à faible intensité (low level laser therapy - LLLT) [9]. Le laser permettrait la stimulation du processus de réparation tissulaire, augmenterait la matrice inorganique osseuse et l'indice mitotique ostéoblastique et stimulerait la croissance des vaisseaux lymphatiques et des capillaires.

- Utilisation de plasma riche en plaquettes (platelet-rich plasma - PRP) [32, 33]

Elle représente $4 \%$ des thérapeutiques expérimentales selon Filleul et al. [29]. Compte tenu de la structure des plaquettes et de leur capacité à interagir avec leur environnement cellulaire, le PRP favorise la cicatrisation. Le fait d'amener directement les plaquettes sur le site permet de créer un environnement local propice à la régénération tissulaire [34]. 
- Ozonothérapie $[9,35]$

Elle représente $12 \%$ des thérapeutiques expérimentales selon Filleul et al. [29]. L'application d'ozone sur l'os nécrosé ou sur les sites d'extraction pendant ou après la réalisation du geste chirurgical invasif permettrait de stimuler la prolifération cellulaire et la cicatrisation tissulaire [9]. Le protocole comporte une antibiothérapie de 15 jours (amoxicilline $2 \mathrm{~g}$ par jour + acide clavulanique, et métronidazole $1 \mathrm{~g}$ par jour) et des séances quotidiennes d'ozonothérapie 7 jours avant et après l'intervention chirurgicale [35].

- Traitement par la parathormone [9]

L'administration à faibles doses de parathormone recombinante (tériparatide ou Forsteo ${ }^{\circledR}$, à $20 \mu \mathrm{g}$ tous les jours en injection sous-cutanée pendant plusieurs mois) augmenterait la densité osseuse et restaurerait la microarchitecture de l'os.

- 0xygénothérapie hyperbare

Elle représente $70 \%$ des techniques expérimentales selon Filleul et al. [29].

\section{Oxygénothérapie hyperbare}

\section{Définition}

L'oxygénothérapie hyperbare $(\mathrm{OHB})$ est une modalité thérapeutique où le patient respire de l'oxygène pur (à $100 \%$ ) à l'intérieur d'une chambre dans laquelle la pression est supérieure à celle du niveau de la mer (atmosphère absolue ou ATA), en général de 1,5 à $3 \mathrm{ATA}$, pendant au moins 90 minutes. Il existe une multitude de protocoles d'administration de l'OHB à des pressions variant de 2 à 3 ATA pour traiter différentes affections [36].

\section{Impact de l'OHB sur les ONM - bases théoriques}

Bien que l'étiopathogénie précise des ONM reste incertaine, certaines propriétés thérapeutiques de l'OHB semblent pouvoir interférer avec le mécanisme de survenue des ONM et justifient, pour certains auteurs, l'utilisation de l'OHB en traitement adjuvant des ONM.

Les données de la littérature concernent les différentes hypothèses étiopathogéniques :

- théorie de l'hypocellularité avec diminution du remodelage et du turn-over osseux

Pour Freiberger, c'est le principal mécanisme sur lequel va agir l'OHB dans le cadre du traitement des ONM [37]. L'OHB s'accompagne d'une augmentation de la néo-formation osseuse [38-41] et du remodelage osseux [42-44] à travers la stimulation d'une puissante protéine recombinante ostéogénique : la rhBMP-2 (recombinant human bone morphogenetic protein-2) [45]. Au total, l'OHB entraînerait une augmentation de la minéralisation et de la densité osseuse [46].

- théorie de l'hypovascularisation

Marx et al. ont montré [42] que l'OHB entraînait une angiogénèse dans le tissu osseux objectivable à partir de 8 séances
d'OHB. Le taux de vascularisation des tissus augmente jusqu'à 20 séances, puis atteint un plateau. Au-delà de 20 séances, l'augmentation du nombre de séances n'impacte plus la densité de la vascularisation. Cette angiogénèse est ensuite permanente puisque 3 ans après le traitement par $\mathrm{OHB}$, la densité de la vascularisation est au moins de $90 \%$ à celle observée juste après la fin des séances d'OHB [43].

- théorie infectieuse

$\mathrm{L}^{\prime} \mathrm{OHB}$ joue à la fois un rôle bactériostatique sur les bactéries anaérobies telles que les Actinomyces et un rôle bactéricide par la production de radicaux libres [44]. Des pressions intermittentes d'oxygène supérieures à 30 à $40 \mathrm{~mm} \mathrm{Hg}$ permettraient d'améliorer l'activité antimicrobienne des leucocytes [47, 48]. En plus d'une action directe sur les bactéries anaérobies, l'OHB potentialiserait l'effet des antibiotiques [48, 49].

- théorie de la toxicité propre des BP et de l'atteinte des tissus épithéliaux buccaux

$\mathrm{L}^{\prime} \mathrm{OHB}$ favoriserait la cicatrisation de la muqueuse [37] dont le potentiel réparateur serait amoindri par l'action des BP.

\section{Résultats de l'utilisation de l'OHB dans le traitement des ONM - revue de littérature}

Depuis 2006, l'OHB a été proposée comme traitement adjuvant des ONM. La plupart de ces études rapportent des cas uniques ou des séries de cas relativement peu importantes dont les résultats sont totalement contradictoires. Selon les publications, on arrive à l'une des conclusions suivantes:

- l'OHB a un effet bénéfique sur le traitement des ONM [2, 7, $22,28,32,37,50-55]$ entrainant une diminution de la taille et du nombre de foyers d'ostéonécrose, et une cicatrisation plus rapide ;

- aucune conclusion n'est possible $[9,31,56,57]$;

- l'OHB n'a aucun impact bénéfique sur les ONM [20, 58-64].

Le protocole utilisé lors des séances d'OHB pour le traitement des ONM n'étant souvent que partiellement décrit par les auteurs $[2,22,28,32,37,50-55]$, la comparaison des résultats obtenus s'avère difficile, voire impossible. Il est à noter que deux essais cliniques contrôlés et randomisés sont actuellement en cours aux Etats-Unis (universités de Duke et du Minnesota) pour évaluer l'intérêt de l'utilisation de l'OHB dans le traitement des ONM $[37,65]$. Les résultats préliminaires de ces études indiquent un effet bénéfique de l'OHB tant sur l'amélioration de la cicatrisation tissulaire que sur la diminution des douleurs à long terme [9].

\section{Discussion}

L'ensemble des études publiées sur l'OHB et les ONM porte exclusivement sur l'évaluation de l'OHB en tant que traitement adjuvant des ONM. Cependant, en l'absence de thérapeutique 
efficace, la prévention doit rester la préoccupation principale de l'ensemble des acteurs de la prise en charge des ONM. Les modalités de cette prévention sont formalisées dans plusieurs recommandations françaises et internationales $[8,9]$. Bien que n'ayant pas fait l'objet de recherche, l'OHB semble pouvoir être un outil à part entière dans la prévention des ONM chez les patients identifiés comme étant à risque de survenue d'une ONM. L'OHB permettrait également, dans les cas peu fréquents où il est possible de retarder le début du traitement par BP, d'accélérer la cicatrisation tissulaire et ainsi de commencer dans un délai plus court le traitement par BP avec un tissu osseux déjà cicatrisé.

Bien que la prévention de la survenue des ONM soit systématique chez les patients traités ou ayant été traités par BP, la définition du patient à risque est importante afin de pouvoir proposer une prise en charge individuelle à chaque patient. Cette définition d'un patient à risque de survenue de ONM repose sur l'analyse des facteurs de risque propres à chaque patient. Parmi ces facteurs de risque, deux sont prépondérants :

- ceux liés au traitement par BP [12] (nature et puissance de la molécule $B P$, voie d'administration, durée du traitement). Il s'agit d'un facteur de risque continu et cumulatif, c'est-à-dire qu'il entraîne une augmentation du risque de survenue de ONM au fil du temps.

- ceux liés à la réalisation d'actes de chirurgie dentaire invasifs tels que les extractions dentaires mais aussi les résections apicales, les actes implantaires, etc. [22, 66-73]. Des études montrent que les actes chirurgicaux (dentaires ou maxillo-faciaux) seraient le facteur déclenchant d'une ONM dans 50 à $88 \%$ des cas [74-77]. Ceci signifie cependant qu'environ 20 à $26 \%$ des ONM apparaissent de manière spontanée [29, 67, 78]. Il s'agit d'un facteur de risque ponctuel, c'est-à-dire qu'un acte chirurgical va augmenter brutalement le risque de survenue d'une ONM en le multipliant en moyenne par $7[9,66]$ (de 5,3 à 21 selon les études $[70,77,79,80])$.

L'OHB n'est pas indiquée en cas de risque continu et cumulatif. L'administration de séances d'OHB sur le long terme à l'ensemble des patients à risque n'aurait pas de sens. Par contre, l'OHB est déjà indiquée en chirurgie orale et maxillo-faciale face à un risque ponctuel identifié, à savoir la prévention de la survenue d'ostéoradionécrose lorsque des extractions dentaires s'imposent dans une zone préalablement irradiée.

À noter qu'il existe actuellement un marqueur sanguin du risque de survenue de ONM mis en évidence par la réalisation du sérum CTx test (C-terminal telopeptide de type I collagen ou ITCP). Il s'agit d'un marqueur de la résorption osseuse qui objective l'élimination de fragments spécifiques produits par l'hydrolyse du collagène de type I [80]. Le risque de survenue de ONM est majoré lorsque le niveau de CTx se situe sous les $150 \mathrm{pg} / \mathrm{ml}$ [66] (100 pg/ml selon une autre étude [81]). Après validation de la valeur du seuil, l'OHB pourrait n'être proposée qu'au patient dont le niveau de CTx se situe en dessous de ce seuil. A noter que d'autres marqueurs biologiques ont été proposés (NTx, PTH, 1,25 dihydroxy vitamin D) [82].

$\mathrm{Au}$ total, deux indications potentielles de l'OHB dans le cadre de la prise en charge des patients traités par BP se dégagent : - en prévention de la survenue de ONM chez les patients identifiés comme étant à risque de survenue d'une ONM lorsque des actes chirurgicaux, dentaires ou maxillo-faciaux s'imposent ;

- en traitement adjuvant des ONM.

Sur la base des travaux publiés et en cours, nous proposons un protocole d'OHB (cf. Tab. II). Le protocole dépend du moment de la prise en charge du patient (prévention ou traitement). Quelle que soit l'évolution de la pathologie, on ne doit pas dépasser 60 séances consécutives d’OHB.

\section{Conclusion}

Le nombre de cas d'ONM ne cessera d'augmenter dans les années à venir du fait notamment de l'utilisation de BP plus puissants, de l'augmentation du nombre de leurs prescriptions, de l'amélioration du dépistage de cette pathologie et du suivi des patients. En raison des conséquences potentiellement délétères des ONM sur la qualité de vie des patients, il apparaît indispensable d'optimiser les moyens de prévention et de traitement des ONM. Pour cela, l'OHB semble être une technique prometteuse quand elle est prescrite chez les patients à risque de survenue d'une ONM lorsque des actes de chirurgie dentaire invasifs s'imposent mais également comme traitement concomitant des ONM, notamment au stade initial de la pathologie. Cependant, des essais cliniques contrôlés et randomisés sont encore nécessaires pour donner un bon niveau de preuve à cette nouvelle indication de l'OHB.

\section{Conflits d'intérêt : aucun}

\section{Références}

1. Smith A, Kressley A, Saif MW. Oral osteonecrosis associated with the use of zoledronic acid: first case with advanced pancreatic cancer and bone metastasis. JOP 2009;10:212-4.

2. Advisory task force on bisphosphonate-related osteonecrosis of the jaws, American association of oral and maxillofacial surgeons. American association of oral and maxillofacial surgeons position paper on bisphosphonate-related osteonecrosis of the jaws. J Oral Maxillofac Surg 2007;65:369-76.

3. Rayman S, Almas K, Dincer E. Bisphosphonate-related jaw necrosis: a team approach management and prevention. Int $J$ Dent Hyg 2009;7:90-5.

4. Marx RE. Oral and intravenous bisphosphonate-induced osteonecrosis of the jaws. History, etiology, prevention, and treatment, $2^{\text {nd }}$ ed. Quintessence Publishing Co, Inc, Chicago, 2007. 
5. Salino S, Bodard AG, Timour Q. Ostéoradionécrose et ostéonécrose des maxillaires aux bisphosphonates. Encyc Med Chir Stomatol 28-865-A-10. Elsevier Masson SAS, Paris, 2010.

6. Wang J, Goodger NM, Pogrel MA. Osteonecrosis of the jaws associated with cancer chemotherapy. J Oral Maxillofac Surg 2003;61:1104-11.

7. Marx RE. Pamidronate (Aredia) and zoledronate (Zometa) induced avascular necrosis of the jaws: a growing epidemic. J Oral Maxillofac Surg 2003;61:1115-7.

8. Agence Nationale de Sécurité des Médicaments et des produits de sante (ANSM). Lettres aux professionnels de santé. Recommandations sur la prise en charge bucco-dentaire des patients traités par bisphosphonates. 18 décembre 2007. Accessible le 21 septembre 2012 sur http://www.ansm.sante.fr/ content/download/11694/140950/version/2/file/lp071203.pdf

9. Ruggiero SL, Dodson TB, Assael LA, Landesberg R, Marx RE, Mehrotra B. American Association of Oral and Maxillofacial Surgeons (AAOMS) position paper on bisphosphonate-related osteonecrosis of the jaw - 2009 update. J Oral Maxillofac Surg 2009;67 (suppl 1):2-12.

10. Abi Najm S, Lesclous P, Lombardi T, Bouzouita I, Carrel JP, Samson J. Ostéonécrose des maxillaires due aux bisphosphonates (2ème partie) : expérience genevoise. Med Buccale Chir Buccale 2008;14:63-84.

11. Savoldelli C, Le Page F, Santini J, Scortecci G, Odin G. Ostéonécrose maxillaire sous bisphosphonates et implants dentaires. Rev Stomatol Chir Maxillofac 2007;108:555-8.

12. Lesclous P, Abi Najm S, Samson J. Bisphosphonates et pratique bucco-dentaire. Rev Odonto-Stomatol 2011;40:173-91.

13. Walter C, Al-Nawas B, Frickhofen N, Gamm H, Beck J, Reinsch L, Blum C, Grötz KA, Wagner W. Prevalence of bisphosphonate associated osteonecrosis of the jaws in multiple myeloma patients. Head Face Med 2010;6:11.

14. Boonyapakorn T, Schirmer I, Reichart PA, Sturm I, Massenkei G. Bisphosphonate-induced osteonecrosis of the jaws: prospective study of 80 patients with multiple myeloma and other malignancies. Oral Oncol 2008;44:857-69.

15. Nalliah R. Prevalence of bisphosphonate-related osteonecrosis in patients with cancer could be as high as 13,3 percent. J Am Dent Assoc 2012;143:170-1.

16. Khan AA, Sandor GK, Dore E. Bisphosphonate associated osteonecrosis of the jaw. J Rheumatol 2009;36:478-90.

17. Khosla S, Burr D, Cauley J, Dempster DW, Ebeling PR, Felsenberg D, Gagel RF, Gilsanz V, Guise T, Koka S, McCauley LK, McGowan J, McKee MD, Mohla S, Pendrys DG, Raisz LG, Ruggiero SL, Shafer DM, Shum L, Silverman SL, Van Poznak CH, Watts N, Woo SB, Shane E; American Society for Bone and Mineral Research. Bisphosphonate-associated osteonecrosis of the jaw: report of a task force of the American society of bone and mineral research. J Bone Miner Res 2007;22:1479-91.

18. Fleish H. Bisphosphonate: mechanisms of action. Endocr Rev 1998;19:80-100.

19. Sedghizadeh PP, Kumar SK, Gorur A, Schaudinn C, Shuler CF, Costerton JW. Identification of microbial biofilms in osteonecrosis of the jaws secondary to bisphosphonate therapy. J Oral Maxillofac Surg 2008;66:767-75.
20. Ruggiero SL, Mehrotra B, Rosenberg TJ, EngroffSL. Osteonecrosis of the jaws associated with the use of bisphosphonate: a review of 63 cases. J Oral Maxillofac Surg 2004;62:527-34.

21. Mashiba T, Hirano T, Turner CH, Forwood MR, Johnston CC, Burr DB. Suppressed bone turnover by bisphosphonates increases microdamage accumulation and reduces some biomechanical properties in dog rib. J Bone Miner Res 2000;15:613-20.

22. Marx RE, Sawatari Y, Fortin M, Broumand V. Bisphosphonateinduces exposed bone (osteonecrosis/osteoporosis) of the jaws: risk factors, recognition, prevention, and treatment. J Oral Maxillofac Surg 2005;63:1567-75.

23. Reid IR, Bolland MJ, Grey AB. Is bisphosphonate-associated osteonecrosis of the jaw caused by soft tissue toxicity? Bone 2007;41:318-20.

24. Reskaa AA, Halasy-Nagy J, Rodan GA. Nitrogen-bisphosphonates block retinoblastoma phosphorylation and cell growth by inhibiting the cholesterol biosynthetic pathway in a keratinocyte model for esophageal irritation. Mol Pharmacol 2001;59:193-202.

25. Brozoski MA, Traina AA, Zindel Deboni MC, Marques MM, NaclérioHomem MG. Bisphosphonate-related osteonecrosis of the jaw. Rev Bras Reumatol 2012;52:265-70.

26. American Dental Association. Dental management of patients receiving oral bisphosphonate therapy: expert panel recommendations. J Am Dent Assoc 2006;137:1144-50.

27. Hillner BE, Ingle JN, Chlebowski RT, Gralow J, Yee GC, Janjan NA, Cauley JA, Blumenstein BA, Albain KS, Lipton A, Brown S; American Society of Clinical Oncology. American Society of Clinical Oncology 2003 update on the role of bisphosphonates and bone health issues in women with breast cancer. J Clin Oncol 2003;21:4042-57. Epub 2003 Sep 8. Erratum in: J Clin Oncol 2004;22:1351.

28. Erkan M, Bilgi 0, Multluoglu M, Uzun G. Bisphosphonate-related osteonecrosis of the jaw in cancer patients and hyperbaric oxygen therapy. JOP. J Pancreas 2009;10:579-80.

29. Filleul 0, Crompot E, Saussez S. Bisphosphonate-induced osteonecrosis of the jaw: a review of 2,400 patient cases. J Cancer Res Clin Oncol 2010;136:1117-24.

30. Vescovi P, Merigo E, Manfredi M. Nd:YAG laser biostimulation in the treatment of bisphosphonate-associated osteonecrosis of the jaw: clinical experience in 28 cases. Photomed Laser Surg 2008;26:37-46.

31. Vescovi P, Manfredi M, Merigo E, Meleti M, Fornaini C, Rocca JP, Nammour S. Surgical approach with ER:YAG Laser on osteonecrosis of the jaws (ONJ in patients under bisphosphonate therapy (BPT). Lasers Med Sci 2010;25:101-13.

32. Antonini F, Pereira CC, Perante EV, Azambuja FG. Management of osteonecrosis of the jaws in patients with history of bisphoshonate therapy. J Craniofac Surg 2010;21:1962-6.

33. Lee YS, David T, Nishime M. Use of platelet-rich plasma in the management of oral bisphonsphonate associated osteonecrosis of the jaw: a report of two cases. J Oral Implantol 2007;26:371-82

34. Eppley BL, Pietrzak WS, Blanton M. Platelet-rich plasma: a review of biology and applications in plastic surgery. Plast Reconstr Surg 2006;118:e147-59. 
35. Agrillo A, Ungari C, Filiaci F, Priore P, Iannetti G. Ozone therapy in the treatment of avascular bisphosphonate-related jaw osteonecrosis. J Craniofac Surg 2007;18:1071-5.

36. Gunepin M, Derache F, Cathelinaud 0, Bladé JS, Risso JJ, Blatteau $\mathrm{JE}$, Bédrune $B$, Hugon $M$, Rivière $D$. Intérêt de l'utilisation de l'oxygénothérapie hyperbare en prévention de l'échec implantaire chez le patient irradié. Med Buccale Chir Buccale 2012;18:14353.

37. Freiberger JJ. Utility of hyperbaric oxygen in treatment of bisphosphonate-related osteonecrosis of the jaws. J Oral Maxillofac Surg 2009;67(suppl 5):96-106.

38. Sawai T, Noomi A, Johansson CB, Sennerby L, Ozeki K, Ozeki K, Takahashi H, Albrektsson T, Ueda M. The effect of hyperbaric oxygen treatment on bone tissue reactions to c.p. titanium implants placed in free autogenous bone grafts. A histomorphometric study in the rabbit mandibule. Clin Oral Implants Res 1998;9:384-97.

39. Tuncay $O C, H o D$, Barker MK. Oxygen tension regulates osteoblast function. Am J Orthod Dentofac Orthop 1994;105:457-63.

40. Granström G. Hyperbaric oxygen as a stimulator of osseointegration. Adv Otorhinolaryngol 1998;54:33-49.

41. Johnsson AA, Sawaii T, Jacobsson M, Granström G, Turesson I. A histomorphometric study of bone reactions to titanium implants in irradiated bone and the effect of hyperbaric oxygen treatment. Int J Oral Maxillofac Implants 1999;14:699-706.

42. Marx RE, Johnson RP, Kline SN. Prevention of osteoradionecrosis: a randomized prospective clinical trial of hyperbaric oxygen versus penicillin. J Am Dent Assoc 1985;111:49-54.

43. Sutthavong $S$, Jansisyanont $P$, Boonyopastham N. Oral health care in head and neck cancer. J Med Assoc Thai 2005; 88 (suppl 3): S339-53.

44. Haute Autorité de Santé (HAS) - service d'évaluation des actes professionnels. Oxygénothérapie hyperbare - janvier 2007 accessible sur http://www.has-sante.fr/portail/upload/docs/ application/pdf/rapport_oxygenotherapie.pdf le 15 septembre 2012.

45. Okubo Y, Bessho K, Fujimura K, Kusumoto K, Ogawa Y, Iizuka T. Effect of hyperbaric oxygenation on bone induced by recombinant human bone morphogenetic protein-2. Br J Oral Maxillofac Surg 2001;39:91-5.

46. Ueng SW, Lee SS, Lin SS, Wang CR, Liu SJ, Yang HF, Tai CL, Shih $\mathrm{CH}$. Bone healing of tibia lengthening is enhanced by hyperbaric oxygen therapy: a study of bone mineral density and torsional strength on rabbits. J Traum 1998;44:676-81.

47. Chen CE, Ko JY, Fu TH, Wang CJ. Results of chronic osteomyelitis of the femur treated with hyperbaric oxygen: a preliminary report. Chang Gung Med J 2004;27:91-7.

48. Tripathi KK, Moorthy A, Karai RC, Rao G, Gosh OC. Effet of hyperbaric oxygen on bone healing after enucleation of mandibular cysts: a modified case-control study. Diving Hyperb Med 2011;41:195-201.

49. Strauss MB, Bryant B. Hyperbaric oxygen. Orthopedics 2002;25:303-10.

50. Ruggiero SL, Woo SB. Bisphosphonate-related osteonecrosis of the jaws. Dent Clin North Am 2008;52:111-28.
51. Wong YK, Cheng JC. Prévention et traitement des ostéonécroses dues aux bisphosphonates : deux cas cliniques. Titane 2009;6:42-7.

52. Freiberger J, Padilla-Burgos R, Chhoeu A, Kraft KH, Boneta 0, Moon RE, Piantadosi CA. Hyperbaric oxygen treatment and bisphosphonate-induces osteonecrosis of the jaw: a case series. J Oral Maxillofac Surg 2007;65:1321-7.

53. Mariotti A. Bisphosphonate and osteonecrosis of the jaws. J Dent Educ 2008;72:919-29.

54. Shirota T, Nakamura A, Matsui Y, Hatori M, Nakamura M, Shintani $S$. Bisphosphonate-related osteonecrosis of the jaw around dental implants in the maxilla: report of a case. Clin Oral Implants Res 2009;20:1402-8.

55. Magopoulos C, Karakinaris G, Telioudis Z, Vahtsevanos K, Dimitrakopoulos I, Antoniadis K, Delaroudis S. Osteonecrosis of the jaws due to bisphosphonate use. A review of 60 cases and treatment proposals. Am J Otolaryngol 2007;28:158-63.

56. Abu-Id MH, Warnke PH, Gottschalk J, Springer I, Wiltfang J, Acil $Y$, Russo PA, Kreusch T. "Bis-phossy jaws" - high and low risk factors for bisphosphonate-induces osteonecrosis of the jaw. J Craniomaxillofac Surg 2008;36:95-103.

57. Lugassy G, Shaham R, Nemets A, Ben-Dor D, Nahlieli 0. Severe osteomyelitis of the jaw in long-term survivors of multiple myeloma: a new clinical entity. Am J Med 2004;117:440-1.

58. Bagan JV, Murillo J, Jimenez Y, Poveda R, Milian MA, Sanchis JM, Silvestre FJ, Scully C. Avascular jaw osteonecrosis in association with cancer chemotherapy: series of 10 cases. J Oral Pathol Med 2005;34:120-3.

59. Ficarra G, Beninati F, Rubino I, Vannucchi A, Longo G, Tonelli P, Pini Prato G. Osteonecrosis of the jaws in periodontal patients with a history of bisphosphonates treatment. J Clin Periodontol 2005;32:1123-8.

60. Farrugia MC, Summerlin DJ, Krowiak E, Huntley T, Freeman S, Borrowdale R, Tomich C. Osteonecrosis of the mandibule or maxilla associated with the use of new generation bisphosphonates. Laryngoscope 2006;116:115-20.

61. Ruggiero SL, Fantasia J, Carlson E. Bisphosphonate-related osteonecrosis of the jaw: background and guidelines for diagnosis, staging and management. Oral Surg Oral Med Oral Pathol Oral Radiol Endod 2006;102:433-41.

62. Chhoeu AH, Longphre JM, Kraft KH, Boneta 0, Conard JL, Freiberger JJ. A case series of hyperbaric oxygen treatment for non-radiation induced osteonecrosis of the jaw. J Oral Maxillofac Surg 2006;64:80-1.

63. Hugentobler M, Richter M. Proposition d'un algorithme de prise en charge médico-dentaire pour les patients traités par bisphosphonates. Rev Stomatol Chir Maxillofac 2006;107;441-4.

64. Magremanne M, Aubert C, Vervaet C, Dufrasne L, Evrard L, Daelemans P. Ostéochimionécrose maxillo-mandibulaire et bisphosphonates. A propos d'un cas et revue de la littérature. Rev Med Brux 2007;28:453-8.

65. Sawatari Y, Marx RE. Bisphosphonates and bisphosphonate induced osteonecrosis. Oral Maxillofac Surg Clin North Am 2007;19:487-98.

66. Marx RE, Cillo JE Jr, Ulloa JJ. Oral bisphosphonate-induced osteonecrosis: risk factors, prediction of risk using serum CTX 
testing, prevention, and treatment. J Oral Maxillofac Surg 2007;65:2397-410.

67. King $A E$, Umland $E M$. Osteonecrosis of the jaw in patient receiving intravenous or oral bisphosphonates. Pharmacotherapy 2008;28:667-77.

68. Pazianas M, Miller P, Blumentals WA, Bernal M, Kothawala P. A review of the literature on osteonecrosis of the jaw in patients with osteoporosis treated with oral bisphosphonates: prevalence, risk factors, and clinical characteristics. Clin Ther 2007;29:1548-58.

69. Estilo CS, Van Poznak CH, Williams T, Evtimovska E, Tkach L, Halpern JL, Tunick SL, Huryn JM. Osteonecrosis of the maxilla and mandible in patients treated with bisphosphonates: a retrospective study. J Clin Oncol 2004;22(suppl 14s):8088.

70. Badros A, Weikel D, Salama A, Goloubeva 0, Schneider A, Rapoport A, Fenton R, Gahres N, Sausville E, Ord R, Meiller T. Osteonecrosis of the jaw in multiple myeloma patients: clinical features and risks factors. J Clin Oncol 2006;24:945-52.

71. Woeller A, Gering A, Brix M, Bettega G, Lebeau J. Ostéonécrose des maxillaires sous bisphosphonates : cinq cas. Rev Stomatol Chir Maxillofac 200;107:417-22.

72. Pautke C, Kreutzer K, Weitz J, Knödler M, Münzel D, Wexel G, Otto S, Hapfelmeier A, Stürzenbaum S, Tischer T. Bisphosphonate related osteonecrosis of the jaw: a minipig large animal model. Bone 2012;51:592-9.

73. Dunstan CR, Felsenberg D, Seibel MJ. Therapy insight: the risks and benefits of bisphosphonates for the treatment of tumorinduced bone disease. Nat Clin Pract Oncol. 2007;4:42-55.

74. Bamias A, Kastritis E, Bamia C, Moulopoulos LA, Melakopoulos I, Bozas G, Koutsoukou V, Gika D, Anagnostopoulos A,
Papadimitriou C, Terpos, E, Dimopoulos MA. Osteonecrosis of the jaw in cancer after treatment with bisphosphonates: incidence and risks factors. J Clin Oncol 2005;23:8580-7.

75. Hoff A0, Toth BB, Altundag K. Osteonecrosis of the jaw in patients receiving intravenous BP therapy. J Clin Oncol 2006;24:8528.

76. Woo SB, Hellstein JW, Kalmar JR. Narrative review: bisphposphonates and osteonecrosis of the jaws. Ann Intern Med 2006;144:753-61.

77. Durie BG, Katz M, Crowley J. Osteonecrosis of the jaw and bisphosphonates. N Engl Med 2005;353:99-102.

78. Bozas G, Roy A, Ramasamy V, Maraveyas A. Osteonecrosis of the jaw after a single bisphosphonate infusion in a patient with metastatic renal cancer treated with sunitinib. Onkologie 2010;33:321-3.

79. Hoff A0, Toth BB, Altundag K, Johnson MM, Warneke CL, Hu M, Nooka A, Sayegh G, Guarneri V, Desrouleaux K, Cui J, Adamus A, Gagel RF, Hortobagyi GN. Frequency and risk factors associated with osteonecrosis of the jaw in cancer patients treated with intravenous bisphosphonates. J Bone Miner Res 2008;23:826-36.

80. Jadu F, Lee L, Pharoah M, Reeve D, Wang L. A retrospective study assessing the incidence, risk factors and comorbidities of pamidronate-related necrosis of the jaws in multiple myeloma patients. Ann Oncol 2007;18:2015-19.

81. Silverman SL, Landesberg R. Osteonecrosis of the jaw and the role of bisphosphonates: a critical review. Am J Med 2009;122 (suppl 2):S33-45.

82. Vescovi P, Nammour S. Bisphosphonate-related osteonecrosis of the jaw (BRONJ) therapy. A critical review. Minerva Stomatol 2010;59:183-203, 204-13. 